\title{
Marchiafava-Bignami disease concurrent with intracerebral hemorrhage: a case description
}

\author{
Zhiwei Zhou ${ }^{1 \#}$, Qinghui Li $^{2 \#}$, Ling Zeng ${ }^{1}$, Tijiang Zhang ${ }^{2}$, Ping Xu ${ }^{1 \wedge}$ \\ ${ }^{1}$ Department of Neurology, Affiliated Hospital of Zunyi Medical University, Zunyi, China; ${ }^{2}$ Department of Radiology, Affiliated Hospital of Zunyi \\ Medical University, Medical Imaging Center of Guizhou Province, Zunyi, China \\ \#These authors contributed equally to this work as co-first authors.
}

Correspondence to: Ping Xu. Department of Neurology, Affiliated Hospital of Zunyi Medical University, Zunyi, China. Email: xuping527@vip.sina.com; Tijiang Zhang. Department of Radiology, Affiliated Hospital of Zunyi Medical University, Medical Imaging Center of Guizhou Province, Zunyi, China. Email: tijzhang@163.com.

Submitted Sep 07, 2021. Accepted for publication Jan 11, 2022.

doi: 10.21037/qims-21-901

View this article at: https://dx.doi.org/10.21037/qims-21-901

\section{Introduction}

Marchiafava-Bignami disease (MBD) is a rare neuropsychiatric disease that is most frequently encountered in people with alcoholism, but also in people who are malnourished $(1,2)$. MBD is pathologically characterized by symmetrical demyelination, necrosis, cystic degeneration, and atrophy of the corpus callosum (CC), usually in its central parts; these characteristics can also be accompanied by extracallosal lesions $(1,2)$. Although the etiology and mechanisms behind MBD remain obscure, its relationships with alcohol abuse and malnutrition suggest that the underlying cause may be metabolic (3). One hypothesis is that alcohol neurotoxicity or nutrient deficiency may contribute to cerebral hypometabolism and acute demyelination of the CC and, in severe cases, lead to cell necrosis (4). Patients with MBD frequently present with unconsciousness, confusion, dysarthria, impaired walking, mutism, and seizures (1).

Intracerebral hemorrhage ( $\mathrm{ICH})$ occurs primarily due to substandard management of hypertension or, less frequently, rupture of a vascular malformation or cerebral amyloid angiopathy (CAA). It is occasionally caused by a hemorrhagic diathesis or cocaine or alcohol abuse (5). Microhemorrhages have been found in the CC, cortex, and subcortex of several patients with MBD (6-10). To our knowledge, ICH has not been observed in a patient with
MBD to date.

This case report presents the first case of a patient with a history of chronic alcoholism presenting with MBD and concurrent ICH in the left external capsule.

\section{Case presentation}

A 58-year-old male was admitted to our hospital (Affiliated Hospital of Zunyi Medical University) with acute onset of slurred speech and slow cognitive response for 1 day. The patient presented with right hemiparesis but had not experienced any headache, nausea, vomiting, mutism, seizures, or incontinence. He had a 30-year history of alcohol abuse and smoking. Before the time of admission, the patient was consuming an average of $500 \mathrm{~mL}$ of Baijiu, a Chinese spirit, per day. Although he did not have a history of hypertension, his blood pressure was 140/92 $\mathrm{mmHg}$ on admission. Confusion was observed, and neurological examinations showed slow cognitive reactions and dysarthria. The patient had normal muscle tone, and the muscle strength in his limbs, was graded as $5 / 5$ on the left and $4 / 5$ on the right, according to the UK Medical Research Council scale. He had a Glasgow Coma Scale (GCS) score of 12 and a National Institute of Health stroke scale (NIHSS) score of 5.

^ ORCID: Zhiwei Zhou, 0000-0001-5499-8740; Ping Xu, 0000-0002-4118-2598. 
An electrocardiogram showed sinus bradycardia, and laboratory findings included the following abnormalities: red blood cell count, $3.64 \times 10^{12} / \mathrm{L}$ [normal range $(\mathrm{NR})$ : 4.3-5.8 $\times 10^{12} / \mathrm{L}$; hematocrit, $0.38 \mathrm{~L} / \mathrm{L}$ (NR: $0.4-0.5 \mathrm{~L} / \mathrm{L}$ ); mean corpuscular volume, $105.5 \mathrm{fL}$ (NR: 80-100 fL); mean corpuscular hemoglobin, $35.7 \mathrm{pg}$ (NR: 27-34 pg); potassium, $3.41 \mathrm{mmol} / \mathrm{L}$ (NR: $3.5-5.3 \mathrm{mmol} / \mathrm{L}$ ); aspartate aminotransferase (AST), $106 \mathrm{U} / \mathrm{L}$ (NR: 15-40 U/L); alkaline phosphatase, $133 \mathrm{U} / \mathrm{L}$ (NR: 45-125 U/L); gamma-glutamyltransferase (GGT), 262 U/L (NR: $10-60 \mathrm{U} / \mathrm{L})$. Other items in the liver function tests [alanine aminotransferase (ALT), bilirubin, and albumin], coagulation tests (international standardized ratio, prothrombin time, prothrombin time activity, activated partial thromboplastin time, fibrinogen, and thrombin time), renal function, myocardial enzyme, blood glucose, and cholesterol were normal. Tests for hepatitis B surface antigen and antibody, hepatitis B e antigen and antibody, hepatitis B core antibody, and hepatitis C virus antibody were negative.

Computed tomography (CT) images of the patient's brain on admission showed a cerebral hemorrhage in the left external capsule (Figure 1A). Magnetic resonance imaging (MRI) was performed on a 3.0 T MR scanner (GE Signa HDxt, Boston, USA) on day 4 after admission, using the following sequences and parameters: axial T1fluid attenuated inversion recovery imaging (FLAIR): repetition time (TR)/time to echo $(\mathrm{TE})=1,420 \mathrm{~ms} / 24 \mathrm{~ms}$, thickness $=5 \mathrm{~mm}$, and slice gap $=6.5 \mathrm{~mm}$; axial T2-weighted imaging (T2WI): (TR/TE $=4,040 \mathrm{~ms} / 118 \mathrm{~ms}$ ); sagittal T2WI: TR/TE $=3,380 \mathrm{~ms} / 118 \mathrm{~ms}$; T2-FLAIR imaging: $\mathrm{TR} / \mathrm{TE}=7,502 \mathrm{~ms} / 145 \mathrm{~ms}$; diffusion-weighted imaging (DWI): TR/TE $=5,175 \mathrm{~ms} / 74.5 \mathrm{~ms}$, field of view $=240 \mathrm{~mm}$ $\times 240 \mathrm{~mm}$, thickness $=5 \mathrm{~mm}$, slice gap $=6.5 \mathrm{~mm}$, and b-value $=1,000$; axial T1-FLAIR contrast: TR/TE $=3,000 \mathrm{~ms} / 25 \mathrm{~ms}$; and susceptibility-weighted imaging (SWI): TR/TE $=41.4 \mathrm{~ms} / 24.96 \mathrm{~ms}$.

The cranial MRI revealed an irregular lesion $(54 \mathrm{~mm} \times$ $20 \mathrm{~mm} \times 27 \mathrm{~mm}$ ) in the center of the left external capsule with hyperintense on axial T1-FLAIR and hypointensity on axial T2WI and T2-FLAIR, surrounded by edema (Figure 1B-1D). The lesions in the central part of the genu, anterior body, and splenium of the CC and those in the bilateral white matter were hyperintense on T2WI and T2-FLAIR, and hypointense on T1-FLAIR (Figure 1B-1D). Gadolinium-enhanced images showed slight enhancement in the hemorrhagic lesion in the left external capsule, but there was no abnormal enhancement in the
CC lesions (Figure 1E). SWI revealed hypointensity of the lesion located in the left external capsule area, as well as multiple microhemorrhages in the brain stem and bilateral basal ganglia, with no vascular malformation (Figure $1 F$ ). Diffusion tensor imaging revealed that the nerve fiber tracts of the left external and internal capsule were interrupted and significantly reduced in number, and a partial reduction in the number of nerve fiber bundles in the CC. Decreased apparent diffusion coefficient (ADC) value in the left external and internal capsule and CC on a color-coded ADC map (Figure 1G). DWI showed restricted diffusion in the CC lesions and a hypointense lesion in the left external capsule (Figure 1H). Sagittal T2WI showed hyperintense lesions in the central part of the genu, anterior body, and splenium of the CC, while the dorsal and ventral layers were not affected (Figure 1I). No obvious abnormalities were observed on head and neck CT angiography. The patient's clinical features combined with his neuroimaging findings confirmed a diagnosis of ICH with MBD.

After diagnosis, the patient was given intravenous multivitamins for MBD and an intramuscular injection of hemocoagulase agkistrodon for conservative treatment of ICH. During hospitalization, the patient's blood pressure fluctuated between 120-130/70-80 mmHg. At discharge, he was conscious and had normal muscle strength, a GCS score of 15 , and an NIHSS score of 0 .

All procedures performed in this study were in accordance with the ethical standards of the Biomedical Research Ethics Committee of Affiliated Hospital of Zunyi Medical University and with the Helsinki Declaration (as revised in 2013). Written informed consent was obtained from the patient for publication of this case report and accompanying images. A copy of the written consent is available for review by the editorial office of this journal.

\section{Discussion}

In the present case, non-contrast head CT failed to detect the lesions in the CC; however, cerebral MRI found symmetric hyperintense lesions in the genu, anterior body, and splenium of the CC on T2WI and T2-FLAIR, with limited diffusion on DWI. Combined with the patient's slurred speech, slow cognitive responses, and history of alcoholism, these results were consistent with a diagnosis of MBD (11). Although previous studies have indicated that head CT can play an important role in the diagnosis of MBD, many patients with MBD are unremarkable on CT but positive on MRI (12). The sensitivity and specificity of 

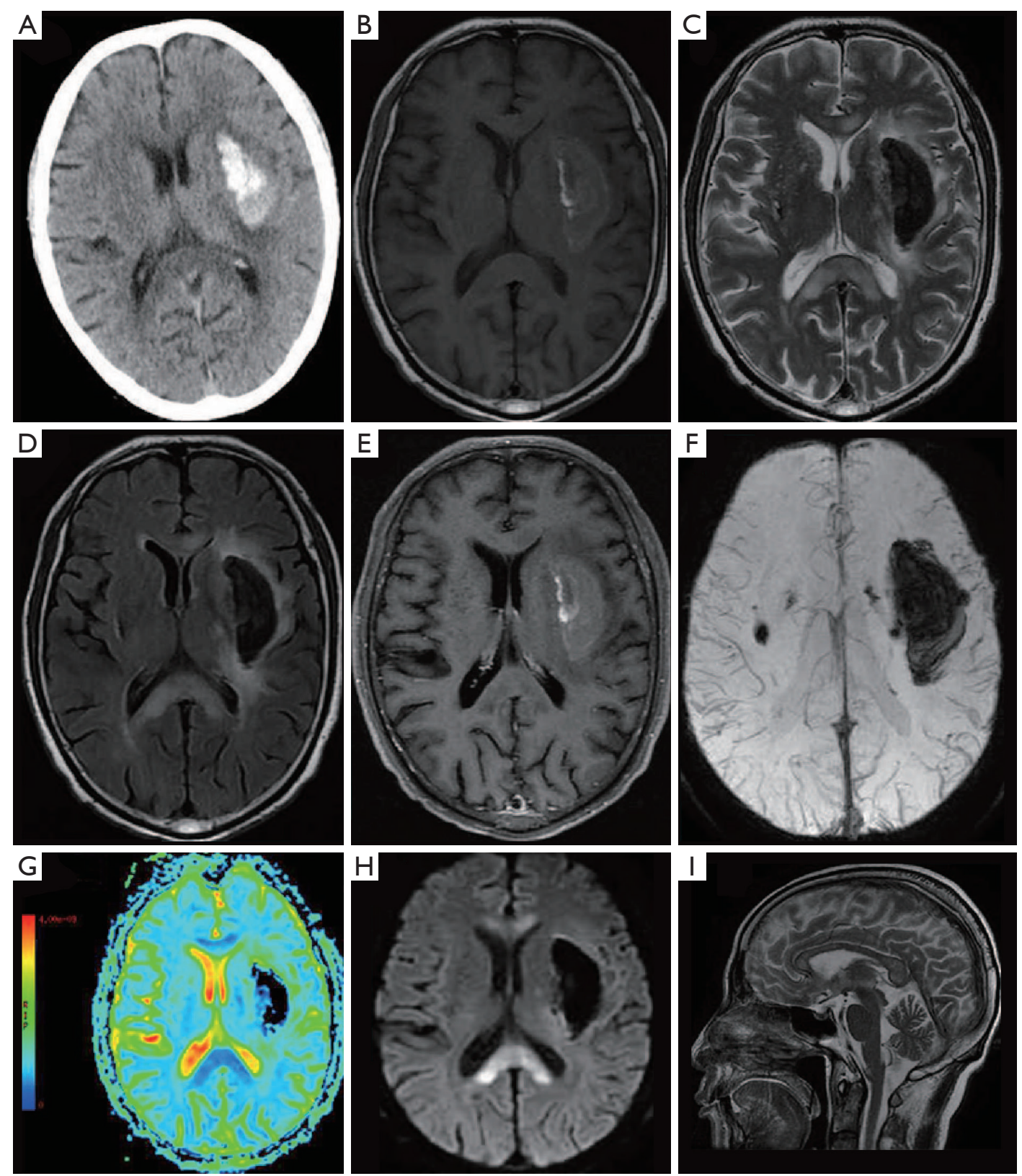

Figure 1 Neuroimages of the patient. (A) Non-contrast head CT image showing a cerebral hemorrhage in the left external capsule. (B,C) An irregular mass in the center of the left external capsule with hyperintense on axial T1-FLAIR and hypointense on axial T2WI, surrounded by edema. The left lateral ventricle, lateral fissure, and some sulcus were compressed and narrowed, and the symmetrical lesions in the CC were hypointense and hyperintense on axial T1-FLAIR and T2WI, respectively. (D) Lesions in the CC and bilateral white matter were hyperintense on T2-FLAIR, and the hemorrhagic lesion was hypointense on T2-FLAIR. (E) Gadolinium-enhanced images showing slight enhancement in the hemorrhagic lesion in the left external capsule, but no abnormal enhancement in the CC. (F) SWI image showing hypointense lesions in the left external capsule area and bilateral basal ganglia. (G) Decreased ADC value in the left external and internal capsule and CC on a color-coded ADC map. (H) DWI image showing restricted diffusion in the lesions of the CC and a hypointense lesion in the left external capsule. (I) Sagittal T2WI image showing hyperintensity in the central part of the genu, anterior body, and splenium of the CC, with the peripheral layers unaffected. CT, computerized tomography; T1-FLAIR, T1-fluid attenuated inversion recovery imaging; T2WI, T2-weighted images; CC, corpus callosum; T2-FLAIR, T2-fluid attenuated inversion recovery imaging; SWI, susceptibilityweighted imaging; ADC, apparent diffusion coefficient; DWI, diffusion-weighted imaging. 
cerebral MRI in diagnosing MBD are therefore superior to those of CT.

Our patient's head CT scan showed a hemorrhagic lesion in the left external capsule. Further SWI showed that there were also several microhemorrhagic lesions in the brain stem and bilateral basal ganglia regions. All of the patient's hemorrhagic lesions, including the major lesion and all microbleeds, were located in the deep brain regions. This observation is consistent with that of a previous study which showed excessive alcohol consumption to be a risk factor for non-lobar ICH, primarily due to its effects on the cerebral small arteries in deep brain regions (13). However, the potential effects of excessive alcohol consumption on vasculopathy have yet to be explored. Excessive alcohol consumption has been associated with the occurrence of ICH at presenium, with heavy drinkers having been found to typically develop ICH between the ages of 52 and 69 years (14). Our patient, who was 58 years old, was within this age range. He did not have a history of hypertension, and his slightly elevated blood pressure on admission was most likely stress-induced.

Because of the considerable influence of chronic alcoholism on the small vessels in the deep brain regions, patients like our patient may have many microhemorrhages in addition to the large hemorrhages shown by head CT scans. We therefore suggest that patients with ICH and a history of alcohol abuse undergo SWI in addition to head $\mathrm{CT}$ and conventional MRI scans.

Our patient presented with elevated AST and GGT, but normal ALT levels, which is consistent with the finding of a previous study that the concentration of AST and GGT is associated with alcohol consumption (15). He also had mildly abnormal liver function and normal coagulation function. Peng et al. found that alcoholism with mild liver disorder may be a warning sign of hemorrhagic stroke in Chinese men and that current smoking is an important independent risk factor for stroke in heavy drinkers (16). Peng et al. also found no significant difference in coagulation function tests, such as prothrombin time and partial thromboplastin time, between heavy drinkers with stroke with and without mild liver disorder (16). Another study also found that alcohol pretreatment had no significant effect on the hematologic system in patients with acute ICH (17).

MBD can be divided into two clinical radiology subtypes according to clinical manifestations and radiologic characteristics: type A (MBD-A) and type B (MBD-B) (2). MBD-A, which is characterized by unconsciousness, pyramidal tract syndromes, and diffuse CC swelling on neuroimaging, has an unfavorable prognosis. The typical features of MBD-B are cognitive impairment, gait disorder, dysarthria, cerebral hemispheric disconnection, and partial callosal lesions, and it is associated with a favorable prognosis. Based on his clinical symptoms and signs, MRI findings, and excellent prognosis, our patient was classified as MBD-B. It has been reported that cognitive impairments in MBD-B may be associated with microhemorrhages, that cerebral microbleeds can worsen cognition, and that there may be an anatomical correlation between the sites of microhemorrhages and the cognitive domains affected (18). Therefore, for timely detection of microhemorrhages and intervention, SWI is highly recommended for patients with MBD-B.

Previous studies on MBD and intraparenchymal hemorrhage have used SWI and CT to detect microbleeds and major hemorrhages. Microbleeds have been reported to occur most frequently in CC lesions and usually in the subacute stage of MBD. Multiple microhemorrhages in the CC may indicate necrosis (6-8). Furthermore, one previous study reported a case in which microbleeds were found in the CC and cortex simultaneously (9), and another reported four cases with microbleeds in both the cortex and subcortex (10). However, the possibility of concurrent CAA or hypertensive cerebral microhemorrhage could not be completely excluded in the study of Kinno et al., considering the higher prevalence rates of these diseases in patients with MBD over the age of 60 years (10).

Our patient did not undergo a brain biopsy to determine whether his multiple microbleeds were related to CAA. However, ICH caused by CAA is usually located in the cerebral lobes, and invasive brain biopsy is not a routine examination in the diagnostic workup of ICH or MBD with progressive improvement. Our patient also sought medical treatment promptly and achieved a favorable outcome, which supports previous findings that the prognosis of patients with MBD who receive early active treatment is superior to those for whom treatment is delayed $(1,19)$.

Alcohol abuse is strongly linked with MBD and is one of the risk factors for cerebrovascular disease. To our knowledge, the present study is the first to report MBD concurrent with ICH in a patient with chronic alcoholism. Further studies are needed to explore whether survivors of MBD are prone to developing concurrent cerebrovascular events. In addition to routine brain CT scans, cerebral MRI and additional SWI are recommended for patients with chronic alcoholism presenting with neuropsychiatric 
abnormalities.

\section{Acknowledgments}

We thank the patient and his family for their collaboration. Funding: None.

\section{Footnote}

Conflicts of Interest: All authors have completed the ICMJE uniform disclosure form (available at https://qims. amegroups.com/article/view/10.21037/qims-21-901/coif). The authors have no conflicts of interest to declare.

Ethical Statement: All procedures performed in this study were in accordance with the ethical standards of the Biomedical Research Ethics Committee of Affiliated Hospital of Zunyi Medical University and with the Helsinki Declaration (as revised in 2013). Written informed consent was obtained from the patient for publication of this case report and accompanying images. A copy of the written consent is available for review by the editorial office of this journal.

Open Access Statement: This is an Open Access article distributed in accordance with the Creative Commons Attribution-NonCommercial-NoDerivs 4.0 International License (CC BY-NC-ND 4.0), which permits the noncommercial replication and distribution of the article with the strict proviso that no changes or edits are made and the original work is properly cited (including links to both the formal publication through the relevant DOI and the license). See: https://creativecommons.org/licenses/by-nc-nd/4.0/.

\section{References}

1. Hillbom M, Saloheimo P, Fujioka S, Wszolek ZK, Juvela $\mathrm{S}$, Leone MA. Diagnosis and management of MarchiafavaBignami disease: a review of CT/MRI confirmed cases. J Neurol Neurosurg Psychiatry 2014;85:168-73.

2. Heinrich A, Runge U, Khaw AV. Clinicoradiologic subtypes of Marchiafava-Bignami disease. J Neurol 2004:251:1050-9.

3. Rickert CH, Karger B, Varchmin-Schultheiss K, Brinkmann B, Paulus W. Neglect-associated fatal Marchiafava-Bignami disease in a non-alcoholic woman. Int J Legal Med 2001;115:90-3.

4. Ishii K, Ikerjiri Y, Sasaki M, Kitagaki H, Mori E.
Regional cerebral glucose metabolism and blood flow in a patient with Marchiafava-Bignami disease. AJNR Am J Neuroradiol 1999;20:1249-51.

5. Jolink WMT, Wiegertjes K, Rinkel GJE, Algra A, de Leeuw FE, Klijn CJM. Location-specific risk factors for intracerebral hemorrhage: Systematic review and metaanalysis. Neurology 2020;95:e1807-18.

6. Boutboul D, Lidove O, Aguilar C, Klein I, Papo T. Marchiafava-Bignami disease complicating SC hemoglobin disease and Plasmodium falciparum infection. Presse Med 2010;39:990-3.

7. Kamaki M, Kawamura M, Moriya H, Hirayama K. Callosal bleeding in a case of Marchiafava-Bignami disease. J Neurol Sci 1996;136:86-9.

8. Shiota JY, Nakano I, Kawamura M, Hirayama K. An autopsy case of Marchiafava-Bignami disease with peculiar chronological CT changes in the corpus callosum: neuroradiopathological correlations. J Neurol Sci 1996;136:90-3.

9. Ishikawa H, Asada R, Shindo A, Suzuki K, Kawamoto E, Fujioka M, Imai H, Tomimoto H. Susceptibility-weighted imaging: Magnetic Resonance Imaging for the detection of microhemorrhages in the corpus callosum of MarchiafavaBignami disease. Neurol Clin Neurosci 2019;7:152.

10. Kinno R, Yamamoto M, Yamazaki T, Owan Y, Fukui T, Kinugasa E. Cerebral microhemorrhage in MarchiafavaBignami disease detected by susceptibility-weighted imaging. Neurol Sci 2013;34:545-8.

11. Yang L, Liu J, Yin Y, Yu H. Simultaneous acute Marchiafava-Bignami disease and posterior reversible encephalopathy syndrome: a case almost misdiagnosed. Quant Imaging Med Surg 2020;10:1392-5.

12. Tung CS, Wu SL, Tsou JC, et al. Marchiafava-Bignami disease with widespread lesions and complete recovery. AJNR Am J Neuroradiol 2010;31:1506-7.

13. Costa P, Grassi M, Iacoviello L, Zedde M, Marcheselli S, Silvestrelli G, et al. Alcohol intake and the risk of intracerebral hemorrhage in the elderly: The MUCHItaly. Neurology 2018;91:e227-35.

14. Casolla B, Dequatre-Ponchelle N, Rossi C, Hénon $\mathrm{H}$, Leys D, Cordonnier C. Heavy alcohol intake and intracerebral hemorrhage: characteristics and effect on outcome. Neurology 2012;79:1109-15.

15. Høiseth G, Hilberg T, Trydal T, Husa A, Vindenes V, Bogstrand ST. The alcohol marker phosphatidylethanol is closely related to AST, GGT, ferritin and HDL-C. Basic Clin Pharmacol Toxicol 2022;130:182-90.

16. Peng GS, Yin SJ, Cheng CA, Chiu SW, Lee JT, Lin WW, 
Lin JC, Hsu YD. Increased risk of cerebral hemorrhage in Chinese male heavy drinkers with mild liver disorder. Cerebrovasc Dis 2007;23:309-14.

17. Peng J, Wang H, Rong X, He L, Xiangpen L, Shen Q, Peng Y. Cerebral Hemorrhage and Alcohol Exposure: A Review. Alcohol Alcohol 2020;55:20-7.

18. Canepa C, Arias L. Partial interhemispheric disconnection syndrome (P-IHDS) secondary to Marchiafava-

Cite this article as: Zhou Z, Li Q, Zeng L, Zhang T, Xu P. Marchiafava-Bignami disease concurrent with intracerebral hemorrhage: a case description. Quant Imaging Med Surg 2022;12(4):2596-2601. doi: 10.21037/qims-21-901
Bignami disease type B (MBD-B). BMJ Case Rep 2016;2016:bcr2016216823.

19. Gambini A, Falini A, Moiola L, Comi G, Scotti G. Marchiafava-Bignami disease: longitudinal MR imaging and MR spectroscopy study. AJNR Am J Neuroradiol 2003;24:249-53. 